\title{
A fixed point theorem in o-minimal structures
}

\author{
Mário J. Edmundo * \\ CMAF Universidade de Lisboa \\ Av. Prof. Gama Pinto 2 \\ 1649-003 Lisboa, Portugal \\ edmundo@cii.fc.ul.pt
}

December 22, 2006

\begin{abstract}
Here we prove an o-minimal fixed point theorem for definable continuous maps on definably compact definable sets, generalizing Brumfiel's version of the Hopf fixed point theorem for semi-algebraic maps.
\end{abstract}

\section{Résumé}

Dans cet article nous montrons un théorème de point fixe o-minimal pour les applications définissables continues sur les ensembles définissables et définissablement compacts, qui généralise la version de Brumfiel du théorème de point fixe de Hopf pour les applications semialgébriques.

${ }^{*}$ Work done while a post-doctoral research fellow at the Mathematical Institute of the University of Oxford and CMAF Universidade de Lisboa with the support of the FCT grant SFRH/BPD/6015/2001. Current support from the FCT (Fundação para a Ciência e Tecnologia), program POCTI (Portugal/FEDER-EU). MSC: 03C64; 55M20. Keywords and phrases: O-minimal structures, fixed point theorems. 


\section{Introduction}

We work over an o-minimal expansion $\mathcal{N}=(N, 0,1,<,+, \cdot, \ldots)$ of a real closed field $N$. Definable means $\mathcal{N}$-definable (possibly with parameters). As it is well known, o-minimal structures are a wide ranging generalization of semi-algebraic and sub-analytic geometry. Good references on o-minimality are, for example, the book [7] by van den Dries and the notes [4] by Michel Coste. For semi-algebraic geometry relevant to this paper the reader should consult the work by Delfs and Knebusch such as [5] and the book [2] by Bochnak, Coste and M-F. Roy.

The goal of the paper is to present the proof of the following o-minimal version of the Lefschetz-Hopf fixed point theorem.

Theorem 1.1 Let $X$ be a definably connected, definably compact definable subset of $N^{n}$. Suppose that $h: X \longrightarrow X$ is a continuous definable map whose Lefschetz number is nonzero. Then $h$ has a fixed point.

Recall that a definable set $X \subseteq N^{n}$ is definably connected if and only if it is not the union of two disjoint clopen definable subsets, and $X$ is definably compact if and only if $X$ is closed and bounded in $N^{n}$ (see [9]).

Most classical proofs of the Lefschetz-Hopf fixed point theorem (see [10]) use repeated barycentric subdivisions and cannot be adapted to the ominimal context since $N$ may be non Archimedean. In [3], Brumfiel proved Theorem 1.1 for the special case of real closed fields. Brumfiel's proof is obtained from the classical proof of the Lefschetz-Hopf fixed point theorem (which works for Archimedean ground fields) by transfer from the field of real algebraic numbers using Tarski's principle. Such arguments fail in our more general context since in general there is no analogue of Tarski's principle.

Our proof of the o-minimal Lefschetz-Hopf fixed point theorem will follow A. Dold's classical proof presented in the book [6]. This approach is based on the theory of the fixed point index which we develop in the o-minimal context. The arguments are exactly the same, we just replace classical topological singular homology by the o-minimal singular homology constructed by $\mathrm{A}$. Woerheide in his thesis [11] and we use a result on definable retractions from [7].

\section{O-minimal homology}

For o-minimal expansions of real closed fields, Woerheide ([11]) constructs o-minimal singular homology $\left(H_{*}, d_{*}\right)$ with coefficients in $\mathbb{Z}$ satisfying ominimal Eilenberg-Steenrod homology axioms (the analogues of the classical Eilenberg-Steenrod axioms for the category of definable sets with definable continuous maps). 
The definition of o-minimal singular homology is quite easy, but the verification of the axioms is very difficult as we now explain. Given a definable set $X$ we consider, for each $m \geq 0$, the abelian group $S_{m}(X)$ freely generated by the singular definable simplices $\sigma: \Delta^{m} \longrightarrow X$, where $\Delta^{m}=\left\{\left(t_{0}, \ldots, t_{m}\right) \in N^{m+1}: \sum_{i} t_{i}=1, t_{i} \geq 0\right\}$ is the standard $m$ dimensional simplex. The boundary operator $\partial_{m+1}: S_{m+1}(X) \longrightarrow S_{m}(X)$ (morphism of degree -1 ) is defined as in the classical case making $S_{*}(X)$ a free chain complex. Also, a definable continuous map $f: X \longrightarrow Y$ induces a chain map $f_{\sharp}: S_{*}(X) \longrightarrow S_{*}(Y)$ (i.e., a morphism of degree zero satisfying $f_{\sharp} \circ \partial_{*}=\partial_{*} \circ f_{\sharp}$ ). Similarly one defines the definable singular chain complex of pairs of definable sets $A \subseteq X$ by $S_{*}(X, A)=S_{*}(X) / S_{*}(A)$. The graded group $H_{*}(X)$ is defined as the homology of the chain complex $S_{*}(X)$. Similarly $H_{*}(X, A)$ is the homology of $S_{*}(X, A)$. A definable continuous map $f: X \longrightarrow Y$ induces a homomorphism $f_{*}: H_{*}(X) \longrightarrow H_{*}(Y)$ of graded groups (via $f_{\sharp}$ ). In the same way, a definable continuous map $f:(X, A) \longrightarrow(Y, B)$ (i.e., a definable continuous map $f: X \longrightarrow Y$ such that $f(A) \subseteq B$ ) induces a homomorphism $f_{*}: H_{*}(X, A) \longrightarrow H_{*}(Y, B)$ of graded groups.

Theorem 2.1 (Woerheide [11]) The o-minimal homology functor $H_{*}$ constructed above satisfies the o-minimal Eilenberg-Steenrod axioms:

Homotopy Axiom. If $f, g:(X, A) \longrightarrow(Y, B)$ are definable maps and there is a definable homotopy between $f$ and $g$, then

$$
f_{*}=g_{*}: H_{n}(X, A) \longrightarrow H_{n}(Y, B)
$$

for all $n \in \mathbb{N}$.

Exactness Axiom. For $A \subseteq X$ definable sets if $i:(A, \emptyset) \longrightarrow(X, \emptyset)$ and $j:(X, \emptyset) \longrightarrow(X, A)$ are the inclusions, then we have a natural exact sequence

$$
\cdots \longrightarrow H_{n}(A, \emptyset) \stackrel{i_{*}}{\rightarrow} H_{n}(X, \emptyset) \stackrel{j_{*}}{\rightarrow} H_{n}(X, A) \stackrel{d_{n}}{\rightarrow} H_{n-1}(A, \emptyset) \longrightarrow \cdots .
$$

Excision Axiom. For every pair $A \subseteq X$ of definable sets and every definable open subset $U$ of $X$ such that $\bar{U} \subseteq \AA$, the inclusion $(X-U, A-$ $U) \longrightarrow(X, A)$ induces isomorphisms

$$
H_{n}(X-U, A-U) \longrightarrow H_{n}(X, A)
$$

for all $n \in \mathbb{N}$.

Dimension Axiom. If $X$ is a one point set, then $H_{n}(X, \emptyset)=0$ for all $n \neq 0$ and $H_{0}(X)=\mathbb{Z}$.

Woerheide's result is based on a definable triangulation theorem ([7]) and on the method of acyclic models from homological algebra and is rather complicated due to the fact that, in arbitrary o-minimal expansions of fields, the 
classical simplicial approximation theorem, the method of repeated barycentric subdivisions and the Lebesgue number property for a standard simplex $\Delta^{n}$ fail.

This construction easily gives, as in the classical case treated in [6] Chapter VI, Section 7, o-minimal singular homology with coefficients in $\mathbb{Q}$. Indeed, if $f: X \longrightarrow Y$ is a definable continuous map, one defines o-minimal singular homology with coefficients in $\mathbb{Q}$ by

$$
H_{m}(X ; \mathbb{Q})=H_{m}\left(S_{*}(X) \otimes \mathbb{Q}\right)
$$

and $f_{*}: H_{m}(X ; \mathbb{Q}) \longrightarrow H_{m}(Y ; \mathbb{Q})$ is the homomorphism induced by $f_{\sharp} \otimes$ id. This o-minimal homology with coefficients in $\mathbb{Q}$ satisfies the corresponding Eilenberg-Steenrod axioms (see [6] Chapter VI, 7.1). We often apply the Universal Coefficient theorem and identify $H_{m}(X) \otimes \mathbb{Q}$ with $H_{m}(X ; \mathbb{Q})$ (and the corresponding $f_{*}$ 's) as $\mathbb{Q}$-vector spaces.

By construction of $\left(H_{*}, d_{*}\right)$, one can also develop the theory of products for o-minimal singular homology in the same way as in the classical case treated in [6] Chapter VI and VII. Here we recall only the o-minimal singular homology cross product since this will be used in the proof of our main result.

If $(X, A),(X, B)$ are pairs of definable sets with $A \subseteq X$ and $B \subseteq X$, then we call $(X ; A, B)$ a definable triad. We say that a definable triad $(X ; A, B)$ is an excisive triad (with respect to $\left(H_{*}, d_{*}\right)$ ) if the inclusion $(A, A \cap B) \longrightarrow$ $(A \cup B, B)$ induces isomorphisms $H_{*}(A, A \cap B) \simeq H_{*}(A \cup B, B)$.

Let $(X, A),(Y, B)$ be pairs of definable sets with $A \subseteq X$ and $B \subseteq Y$. Then we will write $(X, A) \times(Y, B)$ for $(X \times Y, A \times Y \cup X \times B)$.

Theorem 2.2 (Künneth Formula for Homology) Assume that $(X, A)$ and $(Y, B)$ are pairs of definable sets with $A \subseteq X$ and $B \subseteq Y$ such that $(X \times Y ; A \times Y, X \times B)$ is an excisive triad. Then, for all $n \in \mathbb{Z}$, there is an isomorphism

$$
\alpha^{\prime \prime}: \sum_{i+j=n} H_{i}(X, A ; \mathbb{Q}) \otimes H_{j}(Y, B ; \mathbb{Q}) \longrightarrow H_{n}((X, A) \times(Y, B) ; \mathbb{Q}) .
$$

Proof. By [8] Proposition 3.2, we have an o-minimal version of the Eilenberg-Zilber theorem: given definable sets $X$ and $Y$, there are unique (up to chain homotopy) natural chain equivalences $S_{*}(X \times Y) \longrightarrow S_{*}(X) \otimes$ $S_{*}(Y)$ and $S_{*}(X) \otimes S_{*}(Y) \longrightarrow S_{*}(X \times Y)$ inverse to each other. Any such chain map is called an $E Z$ map. Furthermore, the $E Z$ maps are commutative, associative and preserve units as in [6] Chapter VI, $12.1-12.5$. The isomorphism $\alpha^{\prime \prime}$ is defined using the $E Z$ maps as its classical analogue in [6] Chapter VI, Corollary 12.12 in the same purely algebraic way.

The homomorphism $\alpha^{\prime \prime}$ from Theorem 2.2 is called the homology (external) cross product and $\alpha^{\prime \prime}(a \otimes b)$ is denoted $a \times b$. 
Theorem 2.3 The homology cross product satisfies the following properties:

(1) Naturality. $(f \times g)_{*}(\alpha \times \beta)=\left(f_{*} \alpha\right) \times\left(g_{*} \beta\right)$.

(2) Skew-commutativity. $t_{*}(\alpha \times \beta)=(-1)^{\operatorname{deg} \alpha \operatorname{deg} \beta} \beta \times \alpha$ where $t$ : $X \times Y \longrightarrow Y \times X$ commutes factors.

(3) Associativity. $(\alpha \times \beta) \times \gamma=\alpha \times(\beta \times \gamma)$.

(4) Units. $1 \times \alpha=\alpha \times 1=\alpha$.

(5) Stability. $d_{*}(\alpha \times \beta)=i_{1 *}\left(d_{*} \alpha \times \beta\right)+i_{2 *}\left((-1)^{\operatorname{deg} \alpha} \alpha \times d_{*} \beta\right)$ where $\alpha \in H_{i}(X, A ; \mathbb{Q}), \beta \in H_{j}(Y, B ; \mathbb{Q})$ and $i_{1}:(A \times Y, A \times B) \longrightarrow(A \times$ $Y \cup X \times B, A \times B)$ and $i_{2}:(X \times B, A \times B) \longrightarrow(A \times Y \cup X \times B, A \times B)$ are the inclusions.

Proof. The proof of this result is purely algebraic and similar to that of its classical analogue in [6] Chapter VII, Section 2. In fact, these properties are consequences of the corresponding properties for the $E Z$ maps.

\section{O-minimal fixed point theorem}

Below by $\mathbf{S}^{n}$ we denote the $n$-sphere in $N^{n+1}$, where $n>1$. Given $x \in \mathbf{S}^{n}$, consider the exact sequence obtained from the exactness axiom

$$
H_{n}\left(\mathbf{S}^{n}-x\right) \rightarrow H_{n}\left(\mathbf{S}^{n}\right) \rightarrow H_{n}\left(\mathbf{S}^{n}, \mathbf{S}^{n}-x\right) \rightarrow H_{n-1}\left(\mathbf{S}^{n}-x\right) .
$$

Since $\mathbf{S}^{n}-x$ is definably homeomorphic to $N^{n}$ and $H_{k}\left(N^{n}\right)=0$ for all $k>0$, we have that the homomorphism

$$
H_{n}\left(\mathbf{S}^{n}\right) \rightarrow H_{n}\left(\mathbf{S}^{n}, \mathbf{S}^{n}-x\right)
$$

induced by the inclusion is an isomorphism. Since $H_{n}\left(\mathbf{S}^{n}\right) \simeq \mathbb{Z}$, by the Mayer-Vietoris sequence for o-minimal singular homology as in the classical case (see [11]), we call the generator $\zeta$ of $H_{n}\left(\mathbf{S}^{n}\right)$ which corresponds to $1 \in \mathbb{Z}$ the fundamental class of $\mathbf{S}^{n}$ and we denote its image in $H_{n}\left(\mathbf{S}^{n}, \mathbf{S}^{n}-x\right)$ by $\zeta_{x}$.

In what follows below, we assume that $n>1$. This is not a big restriction since definable subsets of $N$ and definable maps between definable subsets of $N$ can be seen in an obvious way as definable subsets of $N^{2}$ and definable maps between definable subsets of $N^{2}$.

Let $V$ be an open definable subset of $N^{n}$ and let $K$ be a definably compact definable subset of $V$. We will canonically and definably identify 
$N^{n}$ with an open definable subset of $\mathbf{S}^{n}$ such that $\mathbf{S}^{n}=N^{n} \cup\{\infty\}$. Hence, $V$ (resp. $K$ ) will be definably identified with an open (resp. definably compact) definable subset of $\mathbf{S}^{n}$ which we will also denote by $V$ (resp. $K$ ).

The fundamental class $\zeta_{K} \in H_{n}(V, V-K)$ around $K$ is the image of $\zeta$ by the homomorphism

$$
H_{n}\left(\mathbf{S}^{n}\right) \longrightarrow H_{n}\left(\mathbf{S}^{n}, \mathbf{S}^{n}-K\right) \simeq H_{n}(V, V-K)
$$

where the first homomorphism is induced by inclusion and the last homomorphism is the excision isomorphism.

Thus $\zeta_{K}$ is independent of $V$ and is characterized by the property that, for every $x \in K$, its image under the homomorphism

$$
H_{n}(V, V-K) \longrightarrow H_{n}(V, V-x)
$$

induced by the inclusion is $\zeta_{x} \in H_{n}(V, V-x) \simeq H_{n}\left(\mathbf{S}^{n}, \mathbf{S}^{n}-x\right)$.

Definition 3.1 Let $V$ be an open definable subset of $N^{n}$ and let $h: V \longrightarrow$ $N^{n}$ be a continuous definable map. Assume that the fixed point set $F_{h}=$ $\{x \in V: h(x)=x\}$ of $h$ is definably compact.

The fixed point index $I_{h}$ of $h$ is the element of $\mathbb{Z}$ such that $(i-h)_{*}\left(\zeta_{F_{h}}\right)=$ $I_{h} \zeta_{0}$ where $(i-h)_{*}: H_{n}\left(V, V-F_{h}\right) \longrightarrow H_{n}\left(N^{n}, N^{n}-0\right), i: V \longrightarrow N^{n}$ is the inclusion and $\zeta_{0} \in H_{n}\left(N^{n}, N^{n}-0\right) \simeq H_{n}\left(\mathbf{S}^{n}, \mathbf{S}^{n}-0\right)$.

For the fixed point index $I_{h}$ of $h$ it is very easy to prove the following properties. In fact, the proofs of the corresponding results in [6] Chapter VII, Section 5 carry over.

Proposition 3.2 Let $V$ be an open definable subset of $N^{n}$ and let $h: V \longrightarrow$ $N^{n}$ be a continuous definable map. Assume that the fixed point set $F_{h}=$ $\{x \in V: h(x)=x\}$ of $h$ is definably compact. Then the following hold:

(1) If $W$ is an open definable subset of $N^{n}$ and $K$ is a definably compact, definable subset of $N^{n}$ such that $F_{h} \subseteq K \subseteq W \subseteq V$, then $(i-h)_{*}\left(\zeta_{K}\right)=$ $I_{h} \zeta_{0}$. In particular, $I_{h}$ is independent of $V$.

(2) If $V=\cup\left\{V_{i}: i=1, \ldots, r\right\}, F_{h}^{i}=\left\{x \in V_{i}: h(x)=x\right\}$ is definably compact, $V_{i}$ is an open definable subset for all $i$, and $F_{h}^{i} \cap F_{h}^{j}=\emptyset$ for $i \neq j$, then $I_{h}=\sum_{i=1}^{r} I_{h_{\mid V_{i}}}$.

(3) If $H: V \times[0,1] \longrightarrow N^{n}$ is a definable homotopy between $h_{0}=H(-, 0)$ : $V \longrightarrow N^{n}$ and $h_{1}=H(-, 1): V \longrightarrow N^{n}$ such that the definable set $\{x \in V: H(x, t)=x$ for some $t \in[0,1]\}$ is definably compact, then $I_{h_{0}}=I_{h_{1}}$. 
Proof. (1) This follows from the fact that the inclusion $(W, W-K) \longrightarrow$ $\left(V, V-F_{h}\right)$ takes $\zeta_{K}$ into $\zeta_{F_{h}}$. (2) By [7] Chapter VI, Lemma 3.5, there are disjoint open definable subsets $W_{1}, \ldots, W_{r}$ such that $F_{h}^{i} \subseteq W_{i} \subseteq V_{i}$ for each $i=1, \ldots, r$. Take $W=\cup\left\{W_{i}: i=1, \ldots, r\right\}$. Using the fact that $H_{n}\left(W, F_{h}\right) \simeq \oplus H_{n}\left(W_{i}, W_{i}-F_{h}^{i}\right), \zeta_{W, F_{h}}=\oplus \zeta_{W_{i}, F_{h}^{i}}$ and (1), the result follows. (3) Follows from the homotopy axiom for o-minimal singular homology.

Note that one can also prove the o-minimal analogues of properties [6] Chapter VII (5.5), (5.7) and (5.9) for the fixed point index. The arguments are exactly the same.

Definition 3.3 Let $X$ be a definable set and let $h: X \longrightarrow X$ be a definable continuous map. The Lefschetz number of $h$ over $\mathbb{Q}$ is defined by

$$
\lambda(h ; \mathbb{Q})=\sum_{i=0}^{\operatorname{dim} X}(-1)^{i} \operatorname{tr}_{\mathbb{Q}}\left(h_{*}\right)_{i},
$$

where $\operatorname{tr}_{\mathbb{Q}}\left(h_{*}\right)_{i}$ is the trace of the $\mathbb{Q}$-module homomorphism

$$
\left(h_{*}\right)_{i}: H_{i}(X ; \mathbb{Q}) \longrightarrow H_{i}(X ; \mathbb{Q}) .
$$

The Euler-Poincaré characteristic $\chi(X)$ of $X$ is by definition

$$
\chi(X)=\sum_{i=0}^{\operatorname{dim} X}(-1)^{i} b_{i}=\lambda\left(\operatorname{id}_{X} ; \mathbb{Q}\right)
$$

where $b_{i}=\operatorname{dim}_{\mathbb{Q}} H_{i}(X ; \mathbb{Q})$ are the Betti numbers of $X$.

Below, we will require another characterization of the Lefschetz number of a continuous definable map. This is obtained in [6] Chapter VII, (6.5).

Fact 3.4 Let $L$ be a graded free $\mathbb{Q}$-module. Let $e: L^{*} \otimes_{\mathbb{Q}} L \longrightarrow \mathbb{Q}$ be the evaluation map given by $e\left(\phi \otimes_{\mathbb{Q}} a\right)=\phi(a)$ where $L^{*}$ is the graded dual $\mathbb{Q}$-module given by $\left(L^{*}\right)_{-i}=\operatorname{Hom}_{\mathbb{Q}}\left(L_{i}, \mathbb{Q}\right)$. Let also $\Theta: L^{*} \otimes_{\mathbb{Q}}$ $L \longrightarrow \operatorname{Hom}_{\mathbb{Q}}(L, L)$ be the homomorphism of graded $\mathbb{Q}$-modules given by $\left[\Theta\left(\phi \otimes_{\mathbb{Q}} a\right)\right](b)=(-1)^{\operatorname{deg} a \operatorname{deg} b} \phi(b) a$. By [6] Chapter VII, Proposition 6.3, $\Theta$ is injective.

If $\beta: L \longrightarrow L$ is an endomorphism of $\mathbb{Q}$-modules of finite rank, then by [6] Chapter VII, Proposition 6.3, we have $\beta \in \operatorname{Im} \Theta$ and the Lefschetz number $\Lambda(\beta)$ of $\beta$ is defined by $\Lambda(\beta)=e \circ \Theta^{-1}(\beta)$.

Let $X$ be a definable set. Then by $[8], H_{*}(X ; \mathbb{Q})$ is a graded free $\mathbb{Q}$ module of finite rank. If $h: X \longrightarrow X$ is a definable continuous map, then by [6] Chapter VII, $(6.5)$, we have $\lambda(h ; \mathbb{Q})=e \circ \Theta^{-1}\left(h_{*}\right)=\Lambda\left(h_{*}\right)$.

The following is an adaptation of the proof of [6] Chapter VII, Proposition 6.6: 
Theorem 3.5 Let $K$ be a definably connected, definably compact definable subset of $N^{n}$ and let $h: K \longrightarrow K$ be a continuous definable map. Then there is a continuous definable map $g: V \longrightarrow N^{n}$ such that $V$ is an open definable subset of $N^{n}$ containing $K, h=g_{\mid K}$, the fixed point set of $g$ is a definably compact definable subset of $K$ and $I_{g}=\lambda\left(g_{\mid K} ; \mathbb{Q}\right)=\lambda(h ; \mathbb{Q})$.

Proof. By [7] Proposition 3.3, let $r: V \longrightarrow K$ be a definable retraction of an open definable neighbourhood $V$ of $K$ in $N^{n}$. Let $j: K \longrightarrow V$ be the inclusion map and let $g=j \circ h \circ r: V \longrightarrow N^{n}$.

Since $K$ is definably compact and $F_{g}=\{x \in K: h(x)=x\}$, we see that $F_{g}$ is a closed, and therefore, a definably compact definable subset of $K$. Since $h=g_{\mid K}$, we have to show that $I_{g}=\lambda\left(g_{\mid K} ; \mathbb{Q}\right)$.

Below, the image of the fundamental class $\zeta_{K}$ under $H_{n}(V, V-K) \longrightarrow$ $H_{n}(V, V-K ; \mathbb{Q})$ will still be denoted by $\zeta_{K}$.

Let $\Delta:(V, V-K) \longrightarrow(V, V-K) \times V, \Delta(x)=(x, x)$ be the diagonal map, $d:(V, V-K) \times K \longrightarrow\left(N^{n}, N^{n}-0\right), d(x, y)=x-y$ the difference map and $\widehat{d}: H_{*}(V, V-K ; \mathbb{Q}) \longrightarrow\left(H_{*}(K ; \mathbb{Q})\right)^{*}$ the unique homomorphism of graded $\mathbb{Q}$-algebras such that $d_{*}=\zeta_{0} \circ e \circ \widehat{d} \otimes_{\mathbb{Q}}$ id where $\zeta_{0}: \mathbb{Q} \longrightarrow$ $H_{*}\left(N^{n}, N^{n}-0 ; \mathbb{Q}\right)$ is unique isomorphism determined by the generator $\zeta_{0}$ of $H_{*}\left(N^{n}, N^{n}-0 ; \mathbb{Q}\right)$. Since $d \circ(\mathrm{id} \times g) \circ \Delta=i-g$, we have $I_{g}=(i-g)_{*}\left(\zeta_{K}\right)=$ $e\left(\left(\widehat{d} \otimes_{\mathbb{Q}} g_{*}\right) \circ \Delta_{*}\left(\zeta_{K}\right)\right)$ i.e., $I_{g}=e\left(a_{g}\right)$ where $a_{g}=\left(\widehat{d} \otimes_{\mathbb{Q}} g_{*}\right) \circ \Delta_{*}\left(\zeta_{K}\right)$. Hence, by Fact 3.4 , we have $I_{g}=\Lambda\left(\Theta\left(a_{g}\right)\right)$.

Let $\Phi=\left(\Phi_{i}\right)_{i \in \mathbb{Z}}: H_{*}(K ; \mathbb{Q}) \longrightarrow H_{*}(V ; \mathbb{Q})$ be the homomorphism of graded $\mathbb{Q}$-algebras given by

$$
\Phi_{i}=\left(\zeta_{0} \times-\right)^{-1} \circ(d \times \mathrm{id})_{*} \circ(\mathrm{id} \times t)_{*} \circ(\Delta \times \mathrm{id})_{*} \circ\left(\zeta_{K} \times-\right)
$$

where $t: V \times K \longrightarrow K \times V, t(x, y)=(y, x)$ and $\zeta_{K} \times-: H_{i}(K ; \mathbb{Q}) \longrightarrow$ $H_{i+n}((V, V-K) \times K ; \mathbb{Q})$ and $\zeta_{0} \times-: H_{i}(V ; \mathbb{Q}) \longrightarrow H_{i+n}\left(\left(N^{n}, N^{n}-0\right) \times\right.$ $V ; \mathbb{Q})$ are the homomorphisms induced by the corresponding homology cross homomorphisms (see Theorem 2.3). Note that we have $\left(\widehat{d} \otimes_{\mathbb{Q}}\right.$ id $\left.\otimes_{\mathbb{Q}} g_{*}\right) \circ$ $\left(\mathrm{id} \otimes_{\mathbb{Q}} t_{*}\right)=\left(\mathrm{id} \otimes_{\mathbb{Q}} s_{*}\right) \circ\left(\widehat{d} \otimes_{\mathbb{Q}} g_{*} \otimes_{\mathbb{Q}}\right.$ id $)$ where $s: K \times K \longrightarrow K \times K$ is given by $s(x, y)=(y, x)$. Furthermore, we also have $g_{*} \circ\left(d_{*} \otimes_{\mathbb{Q}}\right.$ id $)=\left(e \otimes_{\mathbb{Q}}\right.$ id $) \circ$ $\left(\widehat{d} \otimes_{\mathbb{Q}}\right.$ id $\left.\otimes_{\mathbb{Q}} g_{*}\right)$. Thus, using the Künneth formula for o-minimal singular homology (see Theorem 2.2) and Fact 3.4, we see that $\Theta\left(a_{g}\right)=g_{*} \circ \Phi$.

Claim: The definable maps $\phi_{1}, \phi_{2}:(V, V-K) \times K \longrightarrow\left(N^{n}, N^{n}-0\right) \times V$ given by $\phi_{1}(x, y)=(x-y, x)$ and $\phi_{2}(x, y)=(x-y, y)$, induce the same homomorphism in homology, $\phi_{1 *}=\phi_{2 *}$.

Proof of Claim: Let $D=\{(x, y) \in V \times K: x=y\}$ and $U=\{(x, y) \in$ $V \times K: \overline{x y} \subseteq V\}$ where $\overline{x y}$ is the segment from $x$ to $y$. Let $\psi_{1}, \psi_{2}$ : $(V \times K, V \times K-D) \longrightarrow\left(N^{n}, N^{n}-0\right) \times V$ be the definable maps given by $\psi_{1}(x, y)=(x-y, x)$ and $\psi_{2}(x, y)=(x-y, y)$. Let $l:(U, U-D) \longrightarrow$ $(V \times K, V \times K-D)$ and $k:(V, V-K) \times K \longrightarrow(V \times K, V \times K-D)$ be the 
inclusion maps and, for $i=1,2$, let $\mu_{i}:(U, U-D) \longrightarrow\left(N^{n}, N^{n}-0\right) \times V$ be the restriction of $\psi_{i}$. Clearly $\mu_{1}$ is definably homotopic to $\mu_{2}$; hence $\mu_{1 *}=\mu_{2 *}$ by the homotopy axiom for o-minimal singular homology. On the other hand, $U$ is an open definable neighbourhood of $D$ in $V \times K$, hence $l_{*}$ is an isomorphism by the excision axiom for o-minimal singular homology. Therefore, $\psi_{1 *}=\psi_{2 *}$ and so $\phi_{1 *}=\phi_{2 *}$ since $\phi_{i}=\psi_{i} \circ k$.

By definition of $\Phi$ we have $\Phi=\left(\zeta_{0} \times-\right)^{-1} \circ \phi_{1 *} \circ\left(\zeta_{K} \times-\right)$. By the claim above, we also have $\Phi=\left(\zeta_{0} \times-\right)^{-1} \circ \phi_{2 *} \circ\left(\zeta_{K} \times-\right)$. Let $B \subseteq N^{n}$ be a closed ball containing 0 and $K$. Then we have $\phi_{2 *} \circ\left(\zeta_{K} \times-\right)=\phi_{*}^{\prime} \circ\left(\zeta_{K} \times-\right)=$ $\phi_{*}^{\prime \prime} \circ\left(\zeta_{B} \times-\right)$ where $\phi^{\prime}:\left(N^{n}, N^{n}-K\right) \times K \longrightarrow\left(N^{n}, N^{n}-0\right) \times V$ is given by $\phi^{\prime}(x, y)=(x-y, y)$ and $\phi^{\prime \prime}:\left(N^{n}, N^{n}-B\right) \times K \longrightarrow\left(N^{n}, N^{n}-0\right) \times V$ is given by $\phi^{\prime \prime}(x, y)=(x-y, y)$. On the other hand, $\phi^{\prime \prime}$ is definably homotopic to the inclusion $j^{\prime} \times j:\left(N^{n}, N^{n}-B\right) \times K \longrightarrow\left(N^{n}, N^{n}-K\right) \times V$ via the definable homotopy $H:[0,1] \times\left(N^{n}, N^{n}-B\right) \times K \longrightarrow\left(N^{n}, N^{n}-0\right) \times V$ given by $H(t,(x, y))=(x-t y, y)$. Therefore, we have $\zeta_{0} \times \Phi(z)=\phi_{*}^{\prime \prime}\left(\zeta_{B} \times z\right)=$ $\left(j_{*}^{\prime} \times j_{*}\right)\left(\zeta_{B} \times z\right)=j_{*}^{\prime}\left(\zeta_{B}\right) \times j_{*}(z)=\zeta_{0} \times j_{*}(z)$. Hence, $\Phi=j_{*}$ and $\Theta\left(a_{g}\right)=$ $g_{*} \circ \Phi=g_{*} \circ j_{*}=\left(g_{\mid K}\right)_{*}$. From this it follows that $I_{g}=\Lambda\left(\Theta\left(a_{g}\right)\right)=\Lambda\left(\left(g_{\mid K}\right)_{*}\right)$ and, by Fact 3.4 , we get $I_{g}=\lambda\left(g_{\mid K} ; \mathbb{Q}\right)$.

From Theorem 3.5 we immediately obtain Theorem 1.1. Also, since on definably compact definable sets the Euler-Poincaré characteristic coincides with the o-minimal Euler characteristic we get the following solution to a problem from [1]:

Corollary 3.6 Suppose that $f: X \longrightarrow X$ is a definable continuous map definably homotopic to the identity, where $X$ is a definably compact, definable set of o-minimal Euler characteristic $E(X)$ different from zero. Then $f$ has a fixed point.

\section{References}

[1] A. Berarducci and M. Otero Transfer methods for o-minimal topology J. Symbolic Logic 68 (3) (2003) 785-794.

[2] J. Bochnak, M. Coste and M-F. Roy Real algebraic geometry Springer-Verlag 1998.

[3] G. W. Brumfiel A Hopf fixed point theorem for semi-algebraic maps Real algebraic geometry (Rennes, 1991), 163-169, Lecture Notes in Math. 1524, Springer Verlag, Berlin, 1992.

[4] M. Coste An introduction to o-minimal geometry Dip. Mat. Univ. Pisa, Dottorato di Ricerca in Matematica, Istituti Editoriali e Poligrafici Internazionali, Pisa (2000). Available in RAAG preprint server 2000 (http://ihp-raag.org/). 
[5] H. Delfs and M. Knebusch On the homology of algebraic varieties over real closed fields J. reine u.angew. Math. 335 (1982) $122-163$.

[6] A. Dold Lectures on algebraic topology Springer Verlag 1995.

[7] L. van den Dries Tame topology and o-minimal structures Cambridge University Press 1998.

[8] M. Edmundo and M. Otero Definably compact abelian groups J. Math. Logic 4 (2) (2004) 163-180.

[9] Y. Peterzil and C. Steinhorn Definable compacteness and definable subgroups of o-minimal groups J. London Math. Soc. 59 (2) (1999) 769-786.

[10] J. Rotman An introduction to algebraic topology Springer Verlag 1988.

[11] A. Woerheide O-minimal homology PhD. Thesis (1996), University of Illinois at Urbana-Champaign. 